\title{
Sound Velocities of Generalized Lennard-Jones $(n-6)$ Fluids Near Freezing
}

\author{
Sergey Khrapak ${ }^{1,2}$ (1) \\ 1 Bauman Moscow State Technical University, 105005 Moscow, Russia; sergey.khrapak@gmx.de \\ 2 Joint Institute for High Temperatures, Russian Academy of Sciences, 125412 Moscow, Russia
}

check for

updates

Citation: Khrapak, S. Sound

Velocities of Generalized

Lennard-Jones $(n-6)$ Fluids Near

Freezing. Molecules 2021, 26, 1660.

https://doi.org/10.3390/

molecules 26061660

Academic Editor: Andrzej Grzechnik

Received: 15 February 2021

Accepted: 15 March 2021

Published: 16 March 2021

Publisher's Note: MDPI stays neutral with regard to jurisdictional claims in published maps and institutional affiliations.

Copyright: (C) 2021 by the author. Licensee MDPI, Basel, Switzerland. This article is an open access article distributed under the terms and conditions of the Creative Commons Attribution (CC BY) license (https:// creativecommons.org/licenses/by/ $4.0 /)$.

\begin{abstract}
In a recent paper [S. Khrapak, Molecules 25, 3498 (2020)], the longitudinal and transverse sound velocities of a conventional Lennard-Jones system at the liquid-solid coexistence were calculated. It was shown that the sound velocities remain almost invariant along the liquid-solid coexistence boundary lines and that their magnitudes are comparable with those of repulsive softsphere and hard-sphere models at the fluid-solid phase transition. This implies that attraction does not considerably affect the magnitude of the sound velocities at the fluid-solid phase transition. This paper provides further evidence to this by examining the generalized Lennard-Jones $(n-6)$ fluids with $n$ ranging from 12 to 7 and demonstrating that the steepness of the repulsive term has only a minor effect on the magnitude of the sound velocities. Nevertheless, these minor trends are identified and discussed.
\end{abstract}

Keywords: Lennard-Jones systems; generalized $(n-6)$ Lennard-Jones potential; sound velocities; fluid-solid phase transition; longitudinal and transverse collective modes

\section{Introduction}

Sound velocities are important characteristics of a substance. They are directly related to long-wavelength excitations-phonons, which play a crucial role in condensed matter, materials science, and soft matter. Therefore, it is important to understand mechanisms that can affect and regulate sound velocities in various situations.

In simple atomic systems with smooth pairwise interaction potentials, the sound velocities can be expressed as sums over atoms involving the first and second derivatives of the interaction potential (see below). In isotropic situations (in gases and liquids), these sums can also be expressed as integrals involving the radial distribution function $g(r)$. The sound velocities are thus completely determined by the shape of the interaction potential and the atomic structure of the system (which are, of course, interrelated).

There have been indications that the shape of the interatomic interaction potential may not be an important factor for the sound velocities in special regions of the phase diagram. For example, the ratio of the sound to thermal velocity of many liquid metals and metalloids has about the same value $\simeq 10$ at the melting temperature [1-3]. A similar value ( $\simeq 9.5$ ) was reported from experiments with solid argon at the melting temperature [4]. Values close to $\simeq 9$ were reported for solid hydrogen and deuterium along the melting curve [5]. Rosenfeld pointed out that this "quasi-universal" property is also shared by the hard-sphere (HS) model [3]. More recently, it was demonstrated that this property is also exhibited by the purely repulsive soft inverse-power-law (IPL) model in a wide range of IPL exponents [6].

A generalization of the celebrated Lindemann's criterion of melting of classical solids was proposed recently on the basis of statistical mechanics arguments [7]. With this generalized formulation, the expressions of the melting temperature are equivalent in three dimensions (3D) and two-dimensions (2D). Moreover, independently of dimensionality, the melting condition predicts that the ratio of the transverse sound velocity to the thermal 
velocity reaches a quasi-universal value along the melting curve (though the magnitudes can be different in 3D and 2D) $[7,8]$.

Motivated by these observations, it was recently investigated in detail how the longitudinal and transverse sound velocities behave at the liquid-solid phase transition of 3D Lennard-Jones (LJ) systems [9]. It was observed that the sound velocities maintain quasi-universal values along the liquid-solid coexistence boundaries and that their magnitudes are comparable with those of repulsive soft-sphere and hard-sphere models. Hence, it was concluded that attraction does not affect the magnitude of sound velocities at the fluid-solid phase transition. This paper provides further evidence for this conclusion by examining the generalized Lennard-Jones $(n-6)$ fluids with $n$ ranging from 12 to 7 to vary the relative strength of attraction. It will be documented that the steepness of the repulsive term has only a relatively weak effect on the magnitudes of the longitudinal and transverse sound velocities.

In principle, one could directly vary the attractive strength by considering a $(12-n)$ LJ system. However, since the long-range $\propto r^{-6}$ attraction has a physical justification (dispersion forces), while the $\propto r^{-12}$ repulsion is just a convenient mathematical approximation (among other possibilities) for a strong repulsion at short distances, the $(n-6)$ systems are much better investigated. Some of the available results will be used below in this paper.

\section{Materials and Methods}

In this work, the generalized $(n-6)$ LJ potential is considered:

$$
\phi(r)=C_{n} \epsilon\left[(\sigma / r)^{n}-(\sigma / r)^{6}\right],
$$

where

$$
C_{n}=\left(\frac{n}{n-6}\right)\left(\frac{n}{6}\right)^{\frac{6}{n-6}},
$$

so that for $n=12$, we get $C_{12}=4$. Here, $\epsilon$ and $\sigma$ are the energy and length scales and the exponent $n$ determines the steepness of the repulsive term in the potential (1). The reduced pressure, energy, density, and temperature are expressed as $P_{*}=P \sigma^{3} / \epsilon, u_{*}=U / N \epsilon$, $\rho_{*}=\rho \sigma^{3}$, and $T_{*}=T / \epsilon$, respectively.

For a spherically symmetric pairwise interaction potential $\phi(r)$, the instantaneous longitudinal and transverse velocities can be expressed (in 3D) as follows [10-12]

$$
\begin{aligned}
& m c_{l}^{2}=\frac{1}{30}\left\langle\sum_{j}\left[2 r_{j} \phi^{\prime}\left(r_{j}\right)+3 r_{j}^{2} \phi^{\prime \prime}\left(r_{j}\right)\right]\right\rangle, \\
& m c_{t}^{2}=\frac{1}{30}\left\langle\sum_{j}\left[4 r_{j} \phi^{\prime}\left(r_{j}\right)+r_{j}^{2} \phi^{\prime \prime}\left(r_{j}\right)\right]\right\rangle .
\end{aligned}
$$

Here, $c_{l}$ and $c_{t}$ are the instantaneous longitudinal and transverse sound velocities, $m$ is the particle mass, the sums run over all neighbours of a test particle, and primes denote derivatives of the interaction potential with respect to the distance $r$. The averaging $\langle\ldots\rangle$ denotes that we perform summation over all $N$ test particles in the system and then divide the sum by $N$. In isotropic fluids, such an averaging is often replaced by an integral involving the radial distribution function $[10,13,14]:\left\langle\sum_{j}(\ldots)\right\rangle \rightarrow 4 \pi \rho \int(\ldots) r^{2} g(r) d r$, where $g(r)$ is the radial distribution function. For further details regarding the evaluation of sound velocities and their relation to the instantaneous elastic moduli, see Ref. [9].

For a pairwise interaction potential, the excess (over the ideal gas) contributions to the energy, $u_{\mathrm{ex}}$, and pressure, $p_{\mathrm{ex}}$, can be expressed via summations similar to those used above (we omit averaging to keep the notation compact):

$$
u_{\mathrm{ex}}=\frac{1}{2 T} \sum_{j} \phi\left(r_{j}\right), \quad p_{\mathrm{ex}}=-\frac{1}{6 T} \sum_{j} r_{j} \phi^{\prime}\left(r_{j}\right),
$$


where the reduced units are used: $u_{\mathrm{ex}}=U_{\mathrm{ex}} / N T, p_{\mathrm{ex}}=P_{\mathrm{ex}} / \rho T$ (the temperature $T$ is measured in energy units, $\left.k_{\mathrm{B}}=1\right)$. It is obvious then that for the $(n-6)$ LJ potential, we can express the reduced sound velocities in terms of reduced excess energy and pressure. After a simple and straightforward algebra, we obtain

$$
\begin{aligned}
& c_{l}^{2} / v_{T}^{2}=\mathcal{A}_{l}(n) u_{\mathrm{ex}}+\mathcal{B}_{l}(n) p_{\mathrm{ex}}, \\
& c_{t}^{2} / v_{T}^{2}=\mathcal{A}_{t}(n) u_{\mathrm{ex}}+\mathcal{B}_{t}(n) p_{\mathrm{ex}},
\end{aligned}
$$

with the $n$-dependent numerical coefficients

$$
\begin{aligned}
& \mathcal{A}_{l}(n)=-\frac{6 n}{5}, \quad \mathcal{B}_{l}(n)=\frac{n(3 n+1)-114}{5(n-6)} \\
& \mathcal{A}_{t}(n)=-\frac{2 n}{5}, \quad \mathcal{B}_{t}(n)=\frac{n(n-3)-18}{5(n-6)} .
\end{aligned}
$$

The sound velocities in Equations (5) and (6) are expressed in units of thermal velocity, $v_{\mathrm{T}}=\sqrt{T / m}$. Equations (5)-(8) can be considered as a generalization of the Zwanzig and Mountain result for the conventional (12-6) LJ potential. In this special case, the constants are $\mathcal{A}_{l}(12)=-72 / 5, \mathcal{B}_{l}(12)=11, \mathcal{A}_{t}(12)=-24 / 5$, and $\mathcal{B}_{t}(12)=3[9,13]$.

The translation to LJ units is straightforward by recognizing that

$$
\begin{aligned}
& P_{*} \equiv \frac{P \sigma^{3}}{\epsilon}=\frac{\rho T\left(1+p_{\mathrm{ex}}\right) \sigma^{3}}{\epsilon}=\rho_{*} T_{*}\left(1+p_{\mathrm{ex}}\right), \\
& u_{*} \equiv \frac{U}{N \epsilon}=\frac{N T\left(3 / 2+u_{\mathrm{ex}}\right)}{N \epsilon}=T_{*}\left(3 / 2+u_{\mathrm{ex}}\right) .
\end{aligned}
$$

In the present paper, we focus on the fluid boundary of the fluid-solid coexistence (freezing line). In the crystalline state, although the isotropic treatment does not hold anymore, the angle-averaged sound velocities can be introduced in essentially the same way as in fluids. Calculated in this way, the sound velocities of the (12-6) LJ system at the solid coexistence boundary are numerically close to those at the fluid boundary, except for in the vicinity of the triple point (see Figure 4 from Ref. [9]).

\section{Results and Discussion}

The sound velocities of generalized $(n-6)$ LJ fluids under freezing conditions have been evaluated using the freezing parameters $\left(T_{*}, P_{*}\right.$, and $\left.\rho_{*}\right)$ tabulated by Sousa et al. [15] (simple analytical fits for the freezing and melting curves are also available [16], but are not used here). Note that the reduced energy tabulated there corresponds to the potential contribution only, $u_{*}=T_{*} u_{\text {ex }}$. The results are presented in Figure 1.

In Figure 1a, the reduced sound velocities are plotted versus the reduced temperature. The main observations are as follows. For each repulsive exponent $n$, the longitudinal and transverse sound velocities remain practically constant along the freezing curve. The approximate constancy of the transverse sound velocity at the fluid-solid phase transition can be interpreted by employing the Lindemann's melting criterion arguments [17]. In one of the formulations of this famous criterion, the melting condition can be expressed in terms of the instantaneous elastic longitudinal and shear moduli and thus in terms of the longitudinal and transverse sound velocities $[7,18]$. In particular, the condition contains a sum over inverse longitudinal and shear moduli, each multiplied by the number of the corresponding collective modes. Since the shear modulus is smaller than the longitudinal one and there are two shear and one longitudinal mode in 3D systems, the contribution from shear modes dominates (this is known as shear dominance), thus leading to the conclusion that $c_{t} / v_{T}$ should be approximately constant at the fluid-solid phase transition [7]. The approximate constancy of the longitudinal sound velocity can be explained as follows. Although the effective softness of a given $\mathrm{LJ}(n-6)$ potential somewhat varies along the freezing curve [19] (i.e., with $T_{*}$ ), the variation of $c_{l} / v_{T}$ with effective softness 
is rather weak (provided the potential is not too soft and long-ranged), see Figure 1 from Ref. [9]. Thus, the reduced longitudinal sound velocity remains approximately constant for interactions that are not too soft.

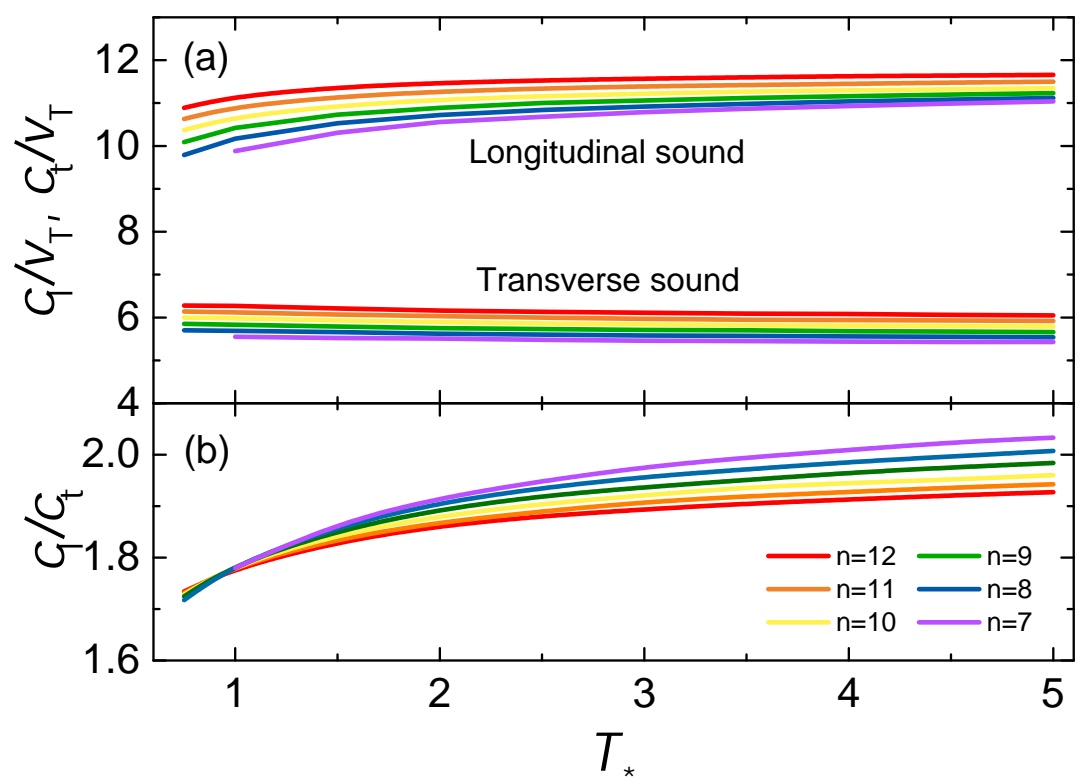

Figure 1. Longitudinal $\left(c_{l}\right)$ and transverse $\left(c_{t}\right)$ sound velocities of generalized LJ $n-6$ fluids at freezing vs the reduced temperature $T_{*}$. In (a) the sound velocities are expressed in units of thermal velocity $v_{\mathrm{T}}$; Upper curves are for the longitudinal mode, lower curves are for the transverse mode. In (b), the ratio of the longitudinal-to-transverse sound velocities, $c_{l} / c_{t}$, is plotted.

Regarding the dependence of the reduced sound velocities on the repulsive exponent $n$ at a fixed $T_{*}$, a very weak, but systematic tendency of increasing $c_{l} / v_{\mathrm{T}}$ and $c_{t} / v_{\mathrm{T}}$ with $n$ is observed. This tendency is consistent with a weak increase in the reduced sound velocities when the HS interaction limit is approached, which has been documented in Figure 1 from Ref. [9]. Increasing $n$ leads to somewhat steeper repulsion and the reduced sound velocities increase accordingly, although very weakly.

To a reasonable approximation, the reduced sound velocities can be approximated by constant values of $c_{l} / v_{\mathrm{T}} \simeq 11$ and $c_{t} / v_{\mathrm{T}} \simeq 6$. Deviations from actual values are within $\sim 10 \%$ for all exponents $n$ in the range of $T_{*}$ considered. The sound velocity of purely repulsive soft inverse-power-law interaction potentials fall within the same range. For instance, the sound velocities of the IPL-12 system at freezing are $c_{l} / v_{\mathrm{T}} \simeq 11.7$ and $c_{t} / v_{\mathrm{T}} \simeq 5.8$ [9]. In this sense, it is legitimate to say that the presence of long-range attraction has a rather minor effect on the sound velocities of simple fluids near the fluid-solid phase transition.

The ratio of the longitudinal-to-transverse sound velocities is plotted in Figure 1b. It exhibits a slow monotonous increase with $T_{*}$. In addition, at higher temperatures, there is an observable decline in $c_{l} / c_{t}$ with increasing $n$. This tendency should be expected. For example, for the potential of soft Yukawa (screened Coulomb) interactions, the ratio $c_{l} / c_{t}$ increases when the interaction becomes softer (screening length increases) and diverges on approaching the one-component plasma limit (see e.g., Figure 2 from Ref. [20]). At the same time, for all $n$ considered here and the range of $T_{*}$ investigated, the ratio $c_{l} / c_{t}$ remains in a relatively narrow range, between $\simeq 1.7$ and $\simeq 2.0$. 


\section{Conclusions}

The instantaneous longitudinal and transverse sound velocities of the generalized $(n-6)$ Lennard-Jones fluids have been evaluated at freezing conditions for integers $n$ between 7 and 12. For this purpose, the relation between the sound velocities (or elastic moduli) and excess energy and pressure derived by Zwanzig and Mountain [13] for the conventional (12-6) LJ potential has been generalized to the case of $(n-6)$ potential. The required freezing densities, pressures, and internal energies for temperatures in the range from $T_{*}=1$ to $T_{*}=5$ have been taken from Ref. [15].

The calculated ratios of sound velocities to the thermal velocity are practically constant along the freezing curves with the typical values $c_{l} / v_{\mathrm{T}} \simeq 11$ and $c_{t} / v_{\mathrm{T}} \simeq 6$ (note that these constant reduced values can result in quite diverse dimensional values when the temperature and the substance atomic mass are varied). These ratios are comparable with reduced sound velocities of repulsive soft sphere (IPL) and HS fluids at freezing. Thus, roughly speaking neither the presence of the long-range attraction nor the variation of the exponent in the repulsive term do not lead to considerable variations in the sound velocities. At the same time, minor systematic trends have been identified and discussed. These trends can be convincingly explained on the basis of the known (weak) dependence of the reduced sound velocities at freezing on the effective softness of the interaction potential.

Funding: Work on the effect of attraction on collective modes in fluids was supported by the Russian Science Foundation, Grant No. 20-12-00356.

Institutional Review Board Statement: Not applicable.

Informed Consent Statement: Not applicable.

Data Availability Statement: This study does not generate any new data.

Conflicts of Interest: The author declares no conflict of interest.

\section{References}

1. Iida, T.; Guthrie, R. The Physical Properties of Liquid Metals; Oxford University Press: New York, NY, USA, 1988.

2. Blairs, S. Sound velocity of liquid metals and metalloids at the melting temperature. Phys. Chem. Liq. 2007, 45, 399-407. [CrossRef]

3. Rosenfeld, Y. Sound velocity in liquid metals and the hard-sphere model. J. Phys. Condens. Matter 1999, 11, L71-L74. [CrossRef]

4. Ishizaki, K.; Spain, I.L.; Bolsaitis, P. Measurements of longitudinal and transverse ultrasonic wave velocities in compressed solidified argon and their relationship to melting theory. J. Chem. Phys. 1975, 63, 1401-1410. [CrossRef]

5. Liebenberg, D.H.; Mills, R.L.; Bronson, J.C. Measurement of P, V, T, and sound velocity across the melting curve of n-H2 and n-D2 to 19 kbar. Phys. Rev. B 1978, 18, 4526-4532. [CrossRef]

6. Khrapak, S.A. Note: Sound velocity of a soft sphere model near the fluid-solid phase transition. J. Chem. Phys. 2016, 144, 126101. [CrossRef] [PubMed]

7. Khrapak, S.A. Lindemann melting criterion in two dimensions. Phys. Rev. Res. 2020, 2, 012040. [CrossRef]

8. Khrapak, S. Note: Melting criterion for soft particle systems in two dimensions. J. Chem. Phys. 2018, 148, 146101. [CrossRef] [PubMed]

9. Khrapak, S.A. Sound Velocities of Lennard-Jones Systems Near the Liquid-Solid Phase Transition. Molecules 2020, $25,3498$. [CrossRef] [PubMed]

10. Schofield, P. Wavelength-dependent fluctuations in classical fluids: I. The long wavelength limit. Proc. Phys. Soc. 1966, 88, 149-170. [CrossRef]

11. Balucani, U.; Zoppi, M. Dynamics of the Liquid State; Clarendon Press: Oxford, UK, 1994.

12. Takeno, S.; Gôda, M. A Theory of Phonons in Amorphous Solids and Its Implications to Collective Motion in Simple Liquids. Prog. Theor. Phys. 1971, 45, 331-352. [CrossRef]

13. Zwanzig, R.; Mountain, R.D. High-Frequency Elastic Moduli of Simple Fluids. J. Chem. Phys. 1965, 43, 4464-4471. [CrossRef]

14. Hubbard, J.; Beeby, J. Collective motion in liquids. J. Phys. C 1969, 2, 556-571. [CrossRef]

15. Sousa, J.M.G.; Ferreira, A.L.; Barroso, M.A. Determination of the solid-fluid coexistence of the $\mathrm{n}-6$ Lennard-Jones system from free energy calculations. J. Chem. Phys. 2012, 136, 174502. [CrossRef] [PubMed]

16. Khrapak, S.A.; Ning, N. Freezing and melting equations for the n-6 Lennard-Jones systems. AIP Adv. 2016, 6, 055215. [CrossRef]

17. Lindemann, F. The calculation of molecular vibration frequencies. Z. Phys. 1910, 11, 609.

18. Buchenau, U.; Zorn, R.; Ramos, M.A. Probing cooperative liquid dynamics with the mean square displacement. Phys. Rev. E 2014, 90, 042312. [CrossRef] [PubMed] 
19. Khrapak, S.A.; Chaudhuri, M.; Morfill, G.E. Communication: Universality of the melting curves for a wide range of interaction potentials. J. Chem. Phys. 2011, 134, 241101. [CrossRef] [PubMed]

20. Khrapak, S.A. Unified description of sound velocities in strongly coupled Yukawa systems of different spatial dimensionality. Phys. Plasmas 2019, 26, 103703. [CrossRef] 